\title{
Development and internal validation of an equation using anthropometric measures to predict correct endotracheal tube insertion depth
}

\author{
Teresa A. Volsko, MBA, MHHS, RRT, CMT-E, FAARC ${ }^{1,2,3}$, Neil L. McNinch, MS, RN PSTAT ${ }^{\circledR 2}$, Christopher Page-Goertz, MD ${ }^{4}$
}

\begin{abstract}
TA Volsko, NL McNinch, C Page-Goertz. Development and internal validation of an equation using anthropometric measures to predict correct endotracheal tube insertion depth. Can J Respir Ther 2022;58:9-14. doi: 10.29390/cjrt-2021-068.

Purpose: To develop, internally validate, and assess the utility of implementing a regression model for determining endotracheal tube (ETT) insertion depth. Research methods: We recorded height, weight, age, sex, ETT internal diameter (ID), lip marking, and tracheal position from the electronic record from a random subset of 2,000 intubated subjects obtained from 1 January 2009 to 5 May 2012. A multivariable linear regression model was constructed and validated by a nonparametric bootstrapping technique using unrestricted random sampling methods. A prospective pilot of subjects admitted to the pediatric intensive care unit requiring invasive mechanical ventilatory support was conducted from 7 January 2019 to 31 May 2019. Those with spinal and/or skeletal malformations, without a post-intubation chest-x-ray (CXR) order, or whose CXR quality impaired visualizing the carina and ETT tip, were excluded. The validated regression equation determined insertion depth. CXR following intubation determined ETT position. Demographic data were summarized. Two-tailed, one-sample binomial test of proportions assessed differences in the proportion of correct position by the equation.

Main findings: Four hundred and seventy-seven subjects included in model construction yielded 10,000 independent samples for internal validation; 55\% were female, and the mean age (SD) was $47(63)$ months. Bias between bootstrap coefficients and refined model estimates were negligible $(P<0.01)$. Eleven subjects in the pilot were female (64\%), mean age (SD) of 36.7 (38) months. Four protocol violations (36.4\%) resulted in malposition. Subsequent repositioning per protocol resulted in $100 \%$ correct positioning $(P=0.01)$.

Conclusion: The regression equation $\left[0.8636 *\left(\mathrm{Ht}^{0}{ }^{0.6223}\right)\right]$ facilitated correct ETT placement. A larger, diverse sample is required for external model validation.
\end{abstract}

Key Words: pediatrics; airway; intubation; malposition; endotracheal tube; radiography

\section{INTRODUCTION}

Airway management is essential to the care of critically ill infants and children. To provide airway protection and invasive ventilatory support, an endotracheal tube (ETT) must be properly placed in the mid-tracheal position [1]. It may be difficult to initially insert and/or maintain the ETT in the mid-tracheal position because of the relatively short length of the trachea in infants and children. The tracheal length ranges from $4 \mathrm{~cm}$ to $6 \mathrm{~cm}$ in infants to $5 \mathrm{~cm}$ to $10 \mathrm{~cm}$ in larger children [2]. Malposition may be further exacerbated by changes in head position, causing the ETT to migrate cauda with neck flexion and cephalad with neck extension [3].

Significant morbidity and mortality are associated with malpositioned ETTs $[4,5]$. Shallowly placed tubes can contribute to unplanned extubation (UE) as well as the improper position of an inflated cuff, when used, between the vocal cords [3]. Malpositioned ETTs leading to UEs often result in emergent endotracheal reintubation, which can be life-threatening if the airway is not secured promptly [6]. Repeated intubations, especially those performed emergently, increase the risk of laryngeal or tracheal injury and scarring [7]. Irritation of the carina and endobronchial intubation may occur when an ETT is placed too deeply [8]. Endobronchial intubations impair ventilation by excessively ventilating one lung and inadequately ventilating the other [8].
To minimize the risk of placing an ETT in a suboptimal position, marks located above the bevel or at the distal end of the ETT provide a crude visual guide for the clinician performing the tracheal intubation. Placing an ETT with the mark just below the vocal cords will theoretically position the tube in the mid-trachea during intubation. The American Academy of Pediatrics Neonatal Resuscitation (NRP) [9] provides guidelines for ETT insertion depth for infants up to 28 days of age to further reduce risk. Depth guidelines were available for infants and children in the 2011 edition of Pediatric Advanced Life Support (PALS) but removed from the current PALS curriculum [10]. Therefore, no guidelines exist for infants over 28 days or pediatric patients. In intubated patients where NRP and former PALS criteria were used to determine ETT insertion depth, 50\% of ETT's in neonates and $41.9 \%$ of ETT's placed in pediatric patients were malpositioned [11, 12]. Underand over-estimated depth of insertion can potentially result in clinically significant consequences. Airway compromise due to UE and mainstem intubation accounted for $23 \%$ of adverse respiratory events among the pediatric population, including death $(41 \%)$ and neurologic damage (21\%) [13].

The literature reports the utility of anthropometric measures or noninvasive quantitative measurements of the body and other external landmarks, such as the sternal notch, as a point of measure when determining

\footnotetext{
${ }^{1}$ Nursing Administration, Akron Children's Hospital, Akron, $\mathrm{OH}$

${ }^{2}$ Rebecca D. Considine Research Institute, Akron Children's Hospital, Akron, $\mathrm{OH}$

${ }^{3}$ The Centers for Families and Children, Cleveland, $\mathrm{OH}$

${ }^{4}$ Division of Critical Care Medicine, Akron Children's Hospital, Akron, $\mathrm{OH}$

Correspondence: Teresa A. Volsko, 4500 Euclid Avenue, Cleveland, OH 44103, United States of America. E-mail: tavolsko@gmail.com
}

Published online at https://www.cjrt.ca on 7 February 2022

This open-access article is distributed under the terms of the Creative Commons Attribution Non-Commercial License (CC BY-NC) (http:// creativecommons.org/licenses/by-nc/4.0/), which permits reuse, distribution and reproduction of the article, provided that the original work is properly cited and the reuse is restricted to noncommercial purposes. For commercial reuse, contact editor@csrt.com 
the correct ETT depth $[14,15]$. The specific aims of this study were to (i) construct and validate a predictive equation, constructed from commonly available anthropomorphic measures to estimate mid-tracheal length in infants and children and (ii) conduct a pilot to determine the utility of implementing the predictive equation in a clinical setting. We hypothesize that using a predictive equation will facilitate the initial placement of the ETT in the mid-tracheal position.

\section{METHODS}

This single-institution study was performed at Akron Children's Hospital, Akron, Ohio. A retrospective chart review was conducted from which the predictive equation was developed, and a protocol for internal validation was conducted. A small prospective study piloted the equation to determine the utility of implementing this new method in a clinical setting. The retrospective and prospective studies were both approved by the institutional review board. A waiver of informed consent was granted for protocol development and internal validation since this portion of the study required no intervention or interaction with the subjects.

\section{Analytical development of the predictive equation}

A retrospective review was conducted of children admitted to the pediatric intensive care unit from 1 January 2009 to 5 May 2012 who required intubation and mechanical ventilatory support. A random subset was selected from these 2,000 subjects who were intubated in the operating room, pediatric intensive care unit, or emergency department with a chest x-ray (CXR) obtained immediately following intubation (Figure 1). The distance from the tip of the ETT to the carina obtained from the radiologist's interpretation, height, weight, age, sex, ETT inner diameter, and centimetre marking at the lip were obtained from the electronic health record. As a standard of care, the depth marking with which the ETT was taped at the lip was recorded in the electronic health record when the CXR was obtained.

We defined proper tracheal position as the ETT tip below the thoracic inlet, identified as the superior border of the clavicular heads and $1 \mathrm{~cm}$ above the carina. In cases where the ETT position was described qualitatively, the radiographic image was retrieved and reviewed by a radiologist blinded to the intent of the request to quantitatively determine and record the position of the ETT. The tolerance level or level of error was determined by the consensus of a group of pediatric intensivists and pediatric radiologists who evaluate imaging and report chest radiographic results at our institution. We defined tolerance level as the difference between predicted and actual distance of the ETT tip to the carina as $\pm 0.25, \pm 0.50$, or $\pm 1.0 \mathrm{~cm}$ for ETTs of $2.5-4 \mathrm{~mm}, 4.5-6.0 \mathrm{~mm}$, or $>6.5 \mathrm{~mm}$ internal diameter (ID), respectively. Poor quality chest radiographs and those with spinal or skeletal deformities were excluded. The data were de-identified and entered in Microsoft Excel (Microsoft, Redmond, CA).

Initial construction of the predictive equation was based on our prior work estimating tracheal length from the superior borders of the clavicular heads to the carina. The predictive equation was initially constructed from three anthropomorphic measures: (i) age, (ii) height, and (iii) sex as covariates [15]; it was then refined to include only height.

\section{Internal validation of the equation}

To begin validation of the equation developed during prior exploratory work, a nonparametric bootstrapping technique was used to validate and refine the equation, using unrestricted random sampling to create independent samples from the data set. These samples were then used to obtain estimates of regression coefficient bias, standard error, and bias-corrected accelerated percentile confidence intervals. Bootstrap resampling techniques are advanced statistical methods known to provide accurate measures of a parameter and do not rely on the normal theory. Bootstrapping resampling techniques are used to create many samples from one, thereby providing a suitable validation sample for testing the performance of a regression equation while minimizing model prediction error [16]. Bootstrapping can also be used to estimate the sampling distribution of an estimator, such as a regression coefficient, bias of the estimator, and confidence intervals for the estimated parameter [16]. The equation was simplified and reconstructed based only upon height. Bootstrapping technique was applied to the simplified equation to determine if the revisions impacted performance.

\section{Sample size determination for prospective pilot}

The $R^{2}$ and effect size of the internally validated predictive equation were used to calculate the minimum sample size for the prospective pilot study. Descriptive statistics were calculated for demographics, guideline adherence, and malposition incidence. The two-tailed binomial test of

\section{FIGURE 1}

Consort diagram of study subjects used in the development and internal validation of the predictive model [7].

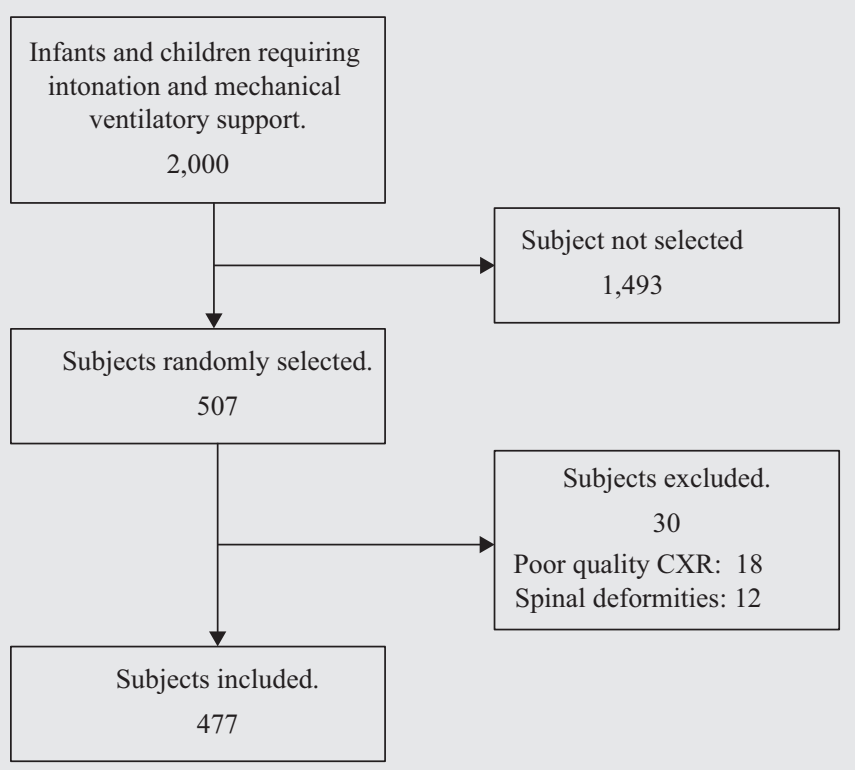


proportions was used to assess the potential difference in the proportion of correct placement using the predictive equation compared with the proportion of correct placement when placement was by predictive equation and/or mark at lip; null proportion $=0.63$.

Statistical analyses were completed using R version 3.1.2 (2014-10-31) and SAS 9.4/14.2. (SAS, Cary, NC). All testing was two-tailed and evaluated at the type I error rate of $P=0.05$ level of statistical significance.

\section{Pilot study methods}

A prospective pilot study of subjects aged $0-18$ years admitted to the pediatric intensive care unit and requiring invasive mechanical ventilatory support from 1 January 2019 to 31 May 2019 was conducted to determine the utility of implementing the equation in the clinical setting to determine ETT insertion depth. Children were excluded from the study if they had spinal and/or skeletal malformations that would hinder the ability to accurately measure the subject's height, did not have a chest radiograph order following intubation, or if the quality of the CXR impaired clear visualization of the superior portion of the clavicular heads, the carina, and ETT.

Before initiating the small prospective pilot, all respiratory therapists scheduled to staff the pediatric intensive care unit were trained on the study protocol and how to access and use the Microsoft Excel spreadsheet to determine ETT insertion depth. The pediatric intensivists reviewed the study protocol during a division meeting. The pediatric intensivists and the respiratory therapists performing the intubation procedure and accessing the preprogrammed spreadsheet were not part of the research team.

The respiratory therapist assigned to the pediatric intensive care unit preparing for intubation used NRP guidelines for tube selection for children up to 28 days of age and the calculation $3.5+$ (age in year/4) to select the appropriately sized cuffed ETT. The respiratory therapist then accessed a preprogrammed Microsoft Excel spreadsheet with the embedded regression equation used to calculate the depth of ETT insertion. The link to the Microsoft Excel spreadsheet was located on a shared drive and accessed through a link on a computerized workstation on wheels. Once the spreadsheet was opened, the respiratory therapist entered the child's height, which was used to complete the following calculation: Lip Mark $=\sim\left[0.8636 *\left(\mathrm{Ht}^{0.6223}\right)\right]$ and provided the centimetre marking the ETT was to be positioned at the lip.

\section{TABLE 1}

Tolerance levels used to determine staff adherence to depth insertion guidelines

\begin{tabular}{lc}
\hline $\begin{array}{l}\text { Endotracheal tube inner } \\
\text { diameter }(\mathbf{m m})\end{array}$ & Tolerance level $(\mathbf{m m})$ \\
\hline $2.5-4.0$ & \pm 2.5 \\
$4.5-6.0$ & \pm 5.0 \\
$\geq 6.5$ & \pm 10.0 \\
\hline
\end{tabular}

The ETT was initially placed at the depth marking predicted by the equation. Immediately following intubation, tracheal placement of the ETT was confirmed by a colorimetric carbon dioxide detector or capnography. Breath sounds and chest wall excursion were assessed as secondary measures, aligned with usual care following endotracheal intubation in the pediatric intensive care unit. If the breath sounds were absent over the left hemithorax or asymmetrical chest rise was noted, the team revalued the tube position, repositioned, and placed a note in the subject's electronic health record.

The first anterior-posterior supine CXR obtained after intubation was used to determine position of the ETT. Children were positioned supine with the head held in a midline position for the imaging. Date of intubation, ETT ID, age of the child in months, height in centimetres, weight in kilograms, sex, calculated marking, depth marking with which the ETT was taped at the lip, and the radiologist determination of the distance from the ETT tip to the carina from the CXR interpretation were collected from the electronic health record. Mirroring the retrospective arm of the study, the depth marking with which the ETT was taped at the lip was recorded in the electronic health record when the CXR was obtained. Calculated marking depth at the lip and the radiologist's determination of the distance from the ETT tip to the carina were used for hypothesis testing. Tolerance levels [12] (Table 1) were used in conjunction with regression model prediction to assess adherence to regression model guidelines.

Following intubation, children remained sedated, and one-to-one nursing care was provided per standard of care in the pediatric intensive care unit. CXR results were available within 10 min of the imaging study. Malpositioned ETTs were immediately repositioned and resecured by the bedside respiratory therapist; depth marking was noted in the electronic medical record, and the provider was notified of the change. This protocol was a longstanding standard of care at the institution and was in place for the retrospective and prospective arms of the study. The researchers debriefed informally with the pediatric intensivists and the respiratory therapists at the conclusion of the study to elicit qualitative factors regarding barriers to the equation's use clinically.

\section{RESULTS}

We reviewed 507 records, 477 of which met inclusion criteria and had complete data for analysis for the retrospective arm of the study. Fifty-five percent of the subjects were female, with a mean (SD) age of $47.0(63.0)$ months.

Spearman's Correlation revealed significant associations with "Superior Clavicle to Carina" distance for age, height, and weight $(r=0.64,0.65$ and 0.69 , respectively, $P<0.01$ for all). Univariate linear regression identified covariates for potential inclusion in a full predictive model (inclusion criteria, $P \leq 0.2$ ). The final significant, nonvalidated linear regression model included age, height, and sex plus their interactions $(P<0.01)$ with an $R^{2}=0.60$. The two full models are shown in Figure 2.

\section{FIGURE 2}

\section{The original non-validated linear regression equations based on height, sex, and age and the refined validated equation.}

Original Model:
Superior Clavicle to Carina distance $\sim 22.248-0.039 *$ Age $+0.135^{*}$
Height $-7.015 *$ Sex $-0.149 *$ Age*Sex $+0.159 *$ Height*Sex $+0.001 *$
Age*Height $+0.001 *$ Age*Height*Sex
$[$ Sex: $1=$ Male, $0=$ Female $]$
Refined Model:
Lip Mark $\sim 0.8636 * \mathrm{Ht}^{0.6223}$




\section{FIGURE 3}

Demographic information from the retrospective cohort of children used to construct the predictive equation and the prospective feasibility and safety pilot.

$I D=$ Internal Diameter.

\begin{tabular}{|c|c|c|c|c|c|c|c|c|c|c|c|c|}
\hline & \multicolumn{6}{|c|}{ Model Building Sample } & \multicolumn{6}{|c|}{ Pilot Study Sample } \\
\hline & $\mathbf{N}$ & $\%$ & Median & IQR & Mean & SD & $\mathbf{N}$ & $\%$ & Median & IQR & Mean & SD \\
\hline Height (cm) & 396 & - & 72.00 & $56-99.5$ & 85.4 & 35.2 & 11 & - & 83.00 & $46.5-134.6$ & 85.2 & 30.1 \\
\hline Weight (kg) & 485 & - & 10.00 & $5.3-18.1$ & 16.8 & 17.2 & 11 & & 11.60 & $5.1-20$ & 15.8 & 13.7 \\
\hline Age (months) & 485 & - & 15.20 & $3.4-59.4$ & 47.0 & 63.0 & 11 & - & 19.00 & $(4-84)$ & 34.5 & 35.7 \\
\hline \multicolumn{13}{|l|}{ Gender } \\
\hline Male & 209 & 45 & - & - & - & - & 4 & 36 & - & - & - & - \\
\hline Female & 252 & 55 & - & - & - & - & 7 & 64 & - & - & - & - \\
\hline \multicolumn{13}{|l|}{ Cuffed ETT ID (mm) } \\
\hline 2.5 & 0 & 0 & - & - & - & - & 0 & 0 & - & - & - & - \\
\hline 3 & 80 & 17 & - & - & - & - & 2 & 18 & - & - & - & - \\
\hline 3.5 & 135 & 29 & - & - & - & - & 2 & 18 & - & - & - & - \\
\hline 4 & 89 & 19 & - & - & - & - & 3 & 27 & - & - & - & - \\
\hline 4.5 & 42 & 9 & - & - & - & - & 0 & 0 & - & - & - & - \\
\hline 5 & 37 & 8 & - & - & - & - & 4 & 36 & - & - & - & - \\
\hline 5.5 & 25 & 5 & - & - & - & - & 0 & 0 & - & - & - & - \\
\hline 6 & 16 & 3 & - & - & - & - & 0 & 0 & - & - & - & - \\
\hline 6.5 & 16 & 3 & - & - & - & - & 0 & 0 & - & - & - & - \\
\hline 7 & 15 & 3 & - & - & - & - & 0 & 0 & - & - & - & - \\
\hline 7.5 & 2 & 0.4 & - & - & - & - & 0 & 0 & - & - & - & - \\
\hline 8 & 4 & 1 & - & - & - & - & 0 & 0 & - & - & - & - \\
\hline
\end{tabular}

Unrestricted random sampling created 10,000 independent samples to validate and refine the equations developed from the retrospective data set. The predictive equation, constructed from age, height, and sex as covariates, had an adjusted $R^{2}=0.60$. The adjusted $R^{2}$ was 0.59 for the simplified equation, using only height. Given the minimal loss in explanation of variance between the original equation and the refined equation, it was deemed prudent to choose the sparse model to avoid over-parameterization and provide a simpler, more utilitarian tool for clinical use. Bias between the bootstrap coefficients and simplified model estimates were negligible $(P<0.01)$, indicating they are stable and appropriately reflect the original sample data structure.

An a priori sample size analysis was conducted prior to the launch of the pilot study. The equation was associated with a large effect size of $f^{2}=1.4$ and a partial correlation of 0.76 ; assuming power $=0.80$ and type I error rate $=0.05$, the minimum estimated sample size needed for the prospective pilot was 11 .

A convenience sample of 11 subjects was included in the feasibility pilot. These patients represented the first 11 patients admitted to the pediatric intensive care unit, whose clinical condition required intubation and mechanical ventilatory support. The cohort was comprised of 7 males $(63.6 \%)$ and 4 females (36.4\%) with a median (IQR) age of 19.0 (4.0-84.0) months. Positioning the ETT at the predicted lip mark was not strictly adhered to in 4 (36.4\%) subjects. In all subjects, once the ETT was positioned and breath sounds assessed, there was no documentation in the electronic health record to substantiate the need to reposition the ETT. No significant oxygen desaturations or UEs occurred during the short period $(\sim 10 \mathrm{~min})$ CXR results were available to the clinical team, and CXR results were available to confirm the tracheal depth of ETT. The ETT in each of these subjects was malpositioned, and subsequent repositioning to the marking at the lip provided by the predictive equation resulted in correct positioning of the ETT in all four subjects $(P=0.01)$. Once repositioned using the equation, the electronic health record documented no evidence of asymmetrical chest movement, or absent breath sounds over the left hemithorax. Figure 3 provides summary data for the subjects enrolled in the retrospective and prospective arms of the study. Table 2 provides a count of the correctly positioned cuffed ETTs by size when the study protocol was followed, and the regression equation was used to determine depth of ETT placement.

\section{DISCUSSION}

Endotracheal intubation is commonly performed across the continuum of care to provide airway protection and invasive mechanical ventilatory support. Achieving and maintaining proper ETT depth is critical to safe and effective clinical care. Significant morbidity and mortality are associated with emergent placement of an ETT improperly positioned in the trachea, which contributes to hypoxemia, pneumothorax, hypotension, cardiac arrhythmias, and the risk for UE [17, 18]. Emergent placement of an ETT following a UE is associated with significant morbidity and mortality [19]. Malpositioned tubes contributing to UE can increase pediatric intensive care and hospital lengths of stay and the cost of care [20]. The literature reports that ETTs are generally placed too deeply when PALS guidelines are used to predict tracheal insertion depth during interfacility transport [21] as well as in the operating room [22], intensive care unit, and the emergency department [23].

Lau et al. [24] modified the age-based formula historically recommended by PALS for children older than 1 year of age and found the modified formula underestimated the depth of insertion in 
TABLE 2

\section{Cuffed ETTs correctly positioned at the lip initially using the regression equation}

\begin{tabular}{lcc}
\hline & $n$ & $\%$ \\
\hline Cuffed ETT ID $(\mathrm{mm})$ size & 7 & 64 \\
$\mathbf{2 . 5}-4.0 / \pm 2.5$ & 6 & 55 \\
$\mathbf{4 . 5}-6.0 / \pm 5.0$ & 1 & 9 \\
$>6.5 / \pm 10.0$ & 0 & 0 \\
\hline
\end{tabular}

Note: $\mathrm{ETT}=$ endotracheal tube; ID = internal diameter

approximately a quarter of children studied. Predictive formulas based on the ETT internal diameter $(3 \times$ ETT ID $\mathrm{mm})$ are limited in use to ETTs with an internal diameter larger than $3 \mathrm{~mm}$ and are associated with malposition rates of $15 \%-25 \%$ [25].

Santos and colleagues [26] evaluated the effectiveness of three different formulas using anthropometric measures to estimate the insertion depth of an endotracheal tube in children in a small cohort of children less than 2 years of age. The formulas based on height measured in $\mathrm{cm}$, $[($ height $/ 10)+5]$, had the strongest correlation $(r=0.88, P<0.05$, concordance correlation coefficient $=0.88$ ) with ideal depth as determined by CXR compared to a weight-based formula or one that used the inner diameter of the ETT [26]. The correlation between the weight-based calculation, with weight measured in $\mathrm{kg}$ (weight +6 ) and depth on CXR, was $r=0.75, P<0.05$, with a concordance correlation coefficient 0.43 , whereas the correlation between endotracheal tube diameter-based calculation and depth on CXR was $r=0.80, P<0.05$, with a concordance correlation coefficient 0.78 [26].

Since the simplified equation was based on prior exploratory work, the bootstrapping technique was determined to be the most suitable method for validating this equation. The simplified predictive equation we tested, based on height, had a predictive adjusted $R^{2}$ of 0.59 , which is similar to the performance in the development cohort. The equation makes use of a measure that can be quickly and easily obtained during an emergency. A prospective pilot was conducted as an intermediate step to determine how practical it was for the clinical team to access and use an equation not tethered to the medical record.

During our prospective pilot study, the equation was accessed by the respiratory therapist and ETT insertion depth determined by the equation; however, the protocol was adhered to in $64 \%(n=7)$ of the subjects. ETTs were correctly positioned within the trachea in all subjects whose physicians agreed with the respiratory therapist's estimation of ETT depth as calculated by the equation in adherence with the study protocol; however, there were four occurrences when the provider preferred the depth of ETT insertion calculated by the guidelines specified in the 2011 edition of PALS [10]. This resulted in study violations and ETT malposition $(n=4,36 \%)$. Although no patient harm (i.e., significant oxygen desaturation, UE) occurred, subsequent repositioning was required. On all four occasions, the physician agreed to use the equation to predict ETT depth, and once the ETT was repositioned to the depth calculated by the equation, correct positioning for all four of these subjects was confirmed. The authors speculate that a greater appreciation of deeply embedded personal biases, such as the preference for how to calculate depth, could have minimized the propensity for protocol violations. The authors acknowledge more attention to exploring preferences and addressing potential causes for protocol violations should have been included in the education and training the respiratory therapists and physicians received.

There were several limitations to this study. The retrospective and prospective arms of the study were conducted in a single institution. The prospective arm or pilot enrolled a very small cohort of subjects. This intermediate step was necessary to evaluate the utility with which a predicted equation would be used clinically. This step was important to our process because it revealed barriers to the practical use of the equation clinically. Although the results are encouraging, they should be interpreted with caution since the small sample size and lack of diversity in the age of subjects enrolled in the study. The four protocol violations illustrate challenges the investigators experienced in garnering support for using the predictive equation by the pediatric intensivists. Comfort with the use of formulas historically recommended by PALS was reported during the debrief session as a barrier to strict adherence to the use of our proposed height-based predictive equation. A larger multi-center trial would be needed to prospectively validate this predictive equation and control for the inherent risk of bias that can occur with a small single institutional study by further removing the investigators from the clinicians conducting the work. Addressing barriers to the use of the equation during our pilot should be considered when educating respiratory therapists and physicians on the study protocol, which will help minimize protocol violations in a larger sample at multiple sites.

The sample of subjects in the retrospective and prospective arms of our research were young (median age 15 months) and reflected the demographics of children admitted to our institution's pediatric intensive care unit. A larger multi-center trial would be necessary to evaluate accuracy of this equation across a more diverse range of ages.

Statistically, the adjusted $R^{2}=0.59$ indicates that there are still unmeasured factors that our studies were unable to identify and contribute to accurate placement. A larger study with a more diverse population may help identify the factors contributing to the accurate estimation of ETT insertion depth.

\section{CONCLUSIONS}

The internally validated model may be useful in determining the depth of ETT insertion in children. Prospective evaluation with a larger sample with more disparate populations (e.g., varying ages) is needed to prospectively determine the value this model has in minimizing ETT malposition following endotracheal intubation.

\section{DISCLOSURES}

\section{Author contributions}

All authors contributed to the protocol development and manuscript preparation. TAV participated in data collection, and NM performed the data analysis.

\section{Conflict of interest}

Teresa A. Volsko declares research grant funding from First Energy Corporation and research relationships with Actuated Medical. Neil McNinch and Christopher Page-Goertz have no relevant financial disclosures.

\section{Financial support}

No financial support was provided for this study.

\section{REFERENCES}

1. Wessels LFA, Reinders MJT, Hart AAM, et al. A protocol for building and evaluating predictors of disease state based on microarray data. Bioinformatics 2005;21:3755-62. doi: 10.1093/bioinformatics/bti429.

2. Griscom NT, Wohl ME. Dimensions of the growing trachea related to age and gender. AJR Am J Roentgenol 1986;146:233-7. doi: 10.2214/ ajr.146.2.233.

3. Sugiyama K, Yokoyama K. Displacement of the endotracheal tube caused by change of head position in pediatric anesthesia: evaluation by fiberoptic bronchoscopy. Anesth Analg 1996;82:251-3. doi: 10.1213/ 00000539-199602000-00006.

4. Niwas R, Nadroo AM, Sutija VG, Gudavalli M, Narula P. Malposition of endotracheal tube: association with pneumothorax in ventilated neonates. Arch Dis Child Fetal Neonatal Ed 2007;92(3):F233-4. doi: 10.1136/adc.2006.106245

5. Simons T, Söderlund T, Handolin L. Radiological evaluation of tube depth and complications of prehospital endotracheal intubation in pediatric trauma: a descriptive study. Eur J Trauma Emerg Surg 2017;43(6):797-804. doi: 10.1007/s00068-016-0758-2.

6. Veldman A, Trautschold T, Weiss K, Fischer D, Bauer K. Characteristics and outcome of unplanned extubation in ventilated pre-term and term newborns on a neonatal intensive care unit. Paediatr Anaesth 2006;16:968-73. doi: 10.1111/j.1460-9592.2006.01902.x. 
7. Abid ES, Miller KA, Monuteaux MC, Nagler J. Association between the number of endotracheal intubation attempts and rates of adverse events in a paediatric emergency department. Emerg Med J 2021. Available at: https://emj.bmj.com/content/early/2021/12/05/emermed-2021-211570 (Accessed January 1, 2022).

8. Mahajan A, Hoftman N, Hsu A, Schroeder R, Wald S. Continuous monitoring of dynamic pulmonary compliance enables detection of endobronchial intubation in infants and children. Anesth Analg 2007;105:51-6. doi: 10.1213/01.ane.0000268119.55909.b4.

9. Weiner GM, ed. Textbook of neonatal resuscitation, 6th ed. Dallas, TX: American Heart Association and American Academy of Pediatrics; 2011.

10. Chameides L, Samson RA, Schexnayder SM, Hazinski MF, eds. Dallas, TX: Pediatric advanced life support: provider manual. American Heart Association and American Academy of Pediatrics; 2011.

11. Phipps LM, Thomas NJ, Gilmore RK, et al. Prospective assessment of guidelines for determining appropriate depth of endotracheal tube placement in children. Pediatr Crit Care Med 2005;6(5):519-22. doi: 10.1097/01.PCC.0000165802.32383.9E.

12. Volsko TA, McNinch NL, Prough DS, Bigham MT. Adherence to endotracheal tube depth guidelines and incidence of malposition in infants and children. Respir Care 2018;63(9):1111-7. doi: 10.4187/respcare.06024.

13. Jimenez N, Posner KL, Cheney FW, Caplan RA, Lee LA, Domino KB. An update on pediatric anesthesia liability: a closed claims analysis. Anesth Analg 2007;104(1):147-53. doi: 10.1213/01.ane.0000246813.04771.03.

14. Locker GJ, Staudinger T, Knapp S, et al. Assessment of the proper depth of endotracheal tube placement with the Trachlight. J Clin Anesth 1998;10(5):389-93. doi: 10.1016/S0952-8180(98)00051-8.

15. Bigham MT, McNinch N, Prough DS, Volsko TA. Use of anthropometric measures to predict tracheal length and correct tracheal tube placement. Respir Care 2017;62(10):OF36.

16. Oredein AI, Olatayo TO, Loyinmi AC. On validating regression models with bootstraps and data splitting techniques. Glob J Sci Frontier Res 2011;11(6): 1-6.

17. Fiadjoe JE, Nishisaki A, Jagannathan N, et al. Airway management complications in children with difficult tracheal intubation from the Pediatric
Difficult Intubation (PeDI) registry: a prospective cohort analysis. Lancet Respir Med 2016;4(1):37-48. doi: 10.1016/S2213-2600(15)00508-1.

18. Powell BM, Gilbert E, Volsko TA. Reducing unplanned extubations in the NICU using lean methodology. Respir Care 2016;61(12):1567-72. doi: $10.4187 /$ respcare. 04540 .

19. Mort TC. Complications of emergency tracheal intubation: immediate airway-related consequences: part II. J Intensive Care Med 2007;22(4):208-15. doi: 10.1177/0885066607301359.

20. Dantin RJ, Spaeder MC, Pastor W, Stockwell DC. Klugman D. Unplanned extubations in children: impact on hospital cost and length of stay. Pediatr Crit Care Med 2015;16(6):572-5. doi: 10.1097/ PCC. 0000000000000406 .

21. Orf J, Thomas SH, Ahmed W, et al. Appropriateness of endotracheal tube size and insertion dept in children undergoing air medical transport. Pediatr Emerg Care 2000;16(5):321-7. doi: 10.1097/00006565200010000-00004.

22. Koshy T, Misra S, Chatterjee N, Dharan BS. Accuracy of a chest x-raybased method for predicting the depth of insertion of endotracheal tubes in pediatric patients undergoing cardiac surgery. J Cardiothorac Vasc Anesth 2016;30(4):947-53. doi: 10.1053/j.jvca.2016.01.031.

23. Harris EA, Arheart KL, Penning DH. Endotracheal tube malposition within the pediatric population: a common event despite clinical evidence of correct placement. Can J Anaesth 2008;55(10): 685-90. doi: 10.1007/BF03017744.

24. Lau N, Playfor SD, Rashad A, Dhanarass M. New formula for predicting tracheal tube length. Paediatr Anaesth 2006;16(12):1238-43. doi: 10.1111/j.1460-9592.2006.01982.x.

25. Phipps LM, Thomas NJ, Gilmore RK, et al. Prospective assessment of guidelines for determining appropriate depth of endotracheal tube placement in children. Pediatr Crit Care Med 2005;6:519-22. doi: 10.1097/01. PCC.0000165802.32383.9E.

26. Santos DLS, Andrade PDO, Gomes ELFD. Does the endotracheal tube insertion depth predicted by formulas in children have a good concordance with the ideal position observed by X-ray? Rev Bras Ter Intensiva 2020;32(2):295-300. doi: 10.5935/0103-507X.20200046. 